\title{
Structure of Turnip mosaic virus and its viral-like particles
}

\author{
Rebeca Cuesta ${ }^{1,3}$, Carmen Yuste-Calvo ${ }^{2,3}$, David Gil-Cartón ${ }^{1}$, Flora Sánchez ${ }^{2}$, \\ Fernando Ponz $\mathbb{D}^{2} \&$ Mikel Valle ${ }^{1 *}$
}

Turnip mosaic virus (TuMV), a potyvirus, is a flexible filamentous plant virus that displays a helical arrangement of coat protein copies (CPs) bound to the ssRNA genome. TuMV is a bona fide representative of the Potyvirus genus, one of most abundant groups of plant viruses, which displays a very wide host range. We have studied by cryoEM the structure of TUMV virions and its viral-like particles (VLPs) to explore the role of the interactions between proteins and RNA in the assembly of the virions. The results show that the CP-RNA interaction is needed for the correct orientation of the CP N-terminal arm, a region that plays as a molecular staple between CP subunits in the fully assembled virion.

Flexible filamentous viruses are plant pathogens that cause important reduction in crop yields and comprise about four hundred different species distributed in four families: Alphaflexiviridae, Betaflexiviridae, Closteroviridae, and Potyviridae (https://viralzone.expasy.org/751). Their virions are long (hundreds of $\mathrm{nm}$ ), thin (about 13-15 $\mathrm{nm}$ in diameter) and flexible, and contain a monopartite $(+)$ ssRNA genome covered by hundreds of subunits of the CP organized in helical fashion ${ }^{1}$. Recent cryoEM studies have solved the structure of several representatives of these helical viruses. The structures for Bamboo mosaic virus (BaMV) ${ }^{2}$ and Pepino mosaic virus (PepMV) ${ }^{3}$, two potexviruses, together with the structure of potyviruses Watermelon mosaic virus (WMV) ${ }^{4}$ and Potato virus $Y(\mathrm{PVY})^{5}$, have shown that these elongated virions display the identical left-handed helical arrangement, and that their CPs share the same fold ${ }^{4,6}$ despite the lack of sequence homology between CPs of viruses from different families.

There is growing interest in the use of plant viruses for nanobiotechnological purposes ${ }^{7}$, specially in biomedical applications where the low potential risk of plant viruses for mammals is a clear advantage ${ }^{8}$. Elongated flexible viruses with helical symmetry can be modified at the level of their CP by genetic engineering or chemical conjugation $^{9}$, and can be used for delivery, imaging, and theranostics purposes. Virions with introduced peptides in their CPs present the antigen in a repetitive and symmetrical way, and it has been shown that they serve as efficient vaccine platforms $s^{10,11}$. Virus like particles (VLPs) devoid of the viral genome are also good nanobiotechnological tools. VLPs of flexible filamentous plant viruses have been produced by the heterologous expression of CPs in bacteria, yeast, insect cells and plants ${ }^{12}$. The biotechnological use of viral nanoparticles (VNPs), which include viruses and VLPs, relies on the successful design of genetic or chemical modifications ${ }^{13}$. Structural information about VLPs from flexible filamentous plant viruses has been scarce ${ }^{14}$, but recently a high resolution study for VLPs from PVY ${ }^{5}$ has shown that the filaments are assembled from octameric rings of the CP, i. e., a nonhelical organization. In this work we explore the structure of TuMV virions and VLPs to unveil the differences in their architecture and understand the contribution of protein-RNA interactions in the assembly of the virions. We observe that TuMV VLPs produced in plants conserve the helical architecture of the virion and that the absence of the ssRNA precludes the interaction between CP subunits mediated by the $\mathrm{N}$-terminal arm.

\section{Results and Discussion}

Using cryoEM and following single particle-based helical image processing, we have explored the structure of the potyvirus TuMV and its VLPs. TuMV virions were isolated from infected plants of Indian mustard, and VLPs of TuMV CP were produced by its transient expression in Nicotiana benthamiana plants ${ }^{15}$. Filaments of virions (Fig. 1a) and VLPs (Fig. 1b) look very similar in cryoEM images, although the VLPs are more variable in length ${ }^{13}$. Extracted segments of the filaments were aligned and classified, and the $2 \mathrm{D}$ averages for TuMV virions and TuMV VLPs are significantly different (insets in Fig. 1). The aligned viral segments display averages with high resolution information with local details attributable to the projection of secondary structural elements of the CPs. The

${ }^{1}$ Molecular Recognition and Host-pathogen Interactions Programme, CIC bioGUNE, Bizkaia Technology Park, 48160, Derio, Spain. ${ }^{2}$ Centro de Biotecnología y Genómica de Plantas, Universidad Politécnica de Madrid - Instituto Nacional de Investigación y Tecnología Agraria y Alimentaria (CBGP, UPM-INIA), Campus Montegancedo, 28223, Madrid, Spain. ${ }^{3}$ These authors contributed equally: Rebeca Cuesta and Carmen Yuste-Calvo. *email: mvalle@ cicbiogune.es 

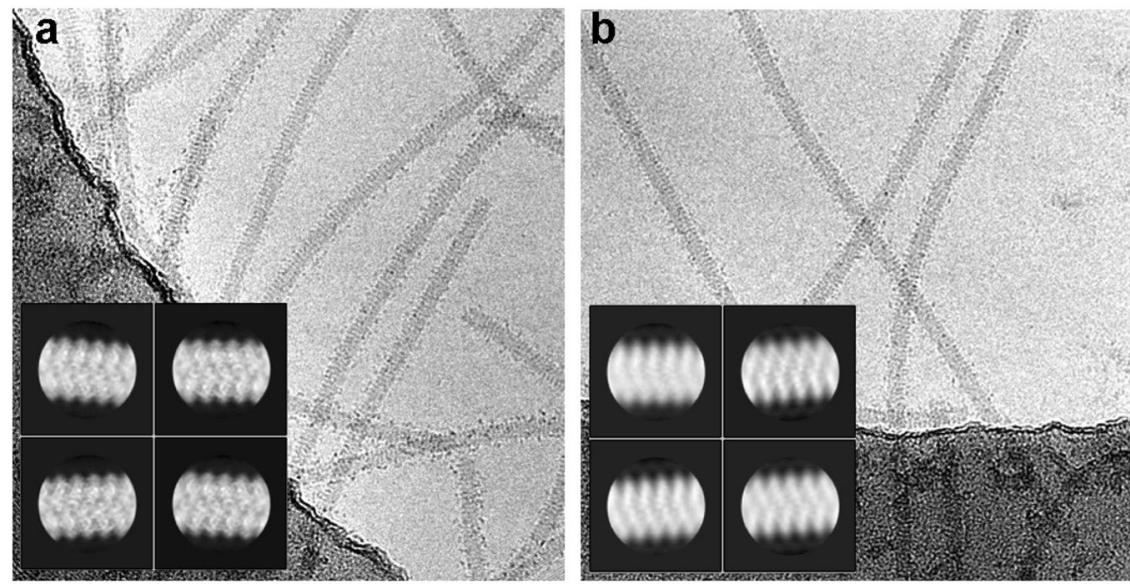

Figure 1. CryoEM imaging of TuMV virions and TuMV VLPs. Panels show cryoEM images for TuMV virions (a) and TuMV VLPs (b). The insets display representative 2D averages for both samples after reference-free classification.

averages from TuMV VLPs, however, are blurred and suggest the presence of structural heterogeneity. These images do not display any pattern of parallel densities, thus, do not suggest TuMV VLPs constructed by stacked rings.

The cryoEM 3D map for TuMV virions (Fig. 2a) shows a left-handed helical arrangement identical to that of earlier characterized flexible filamentous plant viruses ${ }^{2-5}$. Unsupervised 3D classification of the total data set for TuMV virions reveals that regions of the filaments stretch and shrink with an amplitude of around $2 \AA$ per turn (Supplementary Fig. 1a-c and Movie M1). This flexibility of the virions might have limited the resolution which is estimated at approximately $5 \AA$ for the three classes. We have used the $3 \mathrm{D}$ map for the most populated group (Supplementary Fig. 1b) for the calculation of the atomic model for TuMV CP. As mentioned earlier, the $3 \mathrm{D}$ fold of the CPs from flexible filamentous viruses of different families is highly conserved ${ }^{2-4,6}$ despite the absence of sequence homology between them. Within potyviruses the known CP structures for WMV $\mathrm{Wn}^{4} \mathrm{PVY}^{5}$ are almost identical, with rmsd value between $\mathrm{C} \alpha$ atoms around $2 \AA$. The CP from TuMV shows high sequence conservation with these both CPs. Thus, we expect the structure of TuMV to be alike to the structures for the two other potyviruses, WMV and PVY. Actually, the 3D cryoEM maps for TuMV, WMV, and PVY superimpose in almost full agreement (a comparison with WMV is shown in Supplementary Fig. 1e,f). Even though our cryoEM map for TuMV is limited to $5 \AA$ resolution, the high sequence homology and structural conservation allow us to build an accurate atomic model for TuMV CP (Supplementary Fig. 2) based on the structure for WMV CP (pdb code $5 \mathrm{ODV})^{4}$. The sequence homology between the two nucleoproteins is of $63 \%$ identities and $80 \%$ positives in the modeled region. The atomic coordinates for TuMV CP show a central alpha-helical core and two long arms (Fig. 2b). The cryoEM map does not show density (we could not model them) for the first 65 amino acids at the $\mathrm{N}$-terminal end, a flexible region exposed to the solvent. In this regard, cryoEM images for both, virions and VLPs, show small electron-dense bodies around the filaments (Fig. 1) suggesting the presence of partially folded and globular domains in this flexible $\mathrm{N}$-terminus of TuMV CP. The last 16 residues at the C-terminus cannot be traced. As shown before ${ }^{2-4}$, the participation of flexible $\mathrm{N}$ - and $\mathrm{C}$-terminal arms in the interaction between $\mathrm{CP}$ subunits is the structural basis for the flexible nature of the virions. The N-terminal arm of each TuMV CP interacts with other two subunits (Fig. 2a,c). There is a side-by-side interaction between the N-terminal arm and a groove in the adjacent subunit mediated by hydrophobic interactions (Fig. 2c and Supplementary Fig. 2b). After a $90^{\circ}$ turn, the $\mathrm{N}$-terminal arm reaches another subunit in the next turn of the helix where the interaction is favored by complementary electrostatic potentials (Fig. 2c and Supplementary Fig. 2b). The dual role for the N-terminal arm that supports side and axial polymerization and the nature of the local interactions (hydrophobic and electrostatic) were also observed for $\mathrm{WMV}^{4}$ and $\mathrm{PVY}^{5}$ and seem to describe a signature for potyviruses. The density for the ssRNA is clear (red density in Fig. 2d) and each TuMV CP subunit spans five nucleotides of the viral genome. The ssRNA stands in a groove at the folded central domain, just next to the last helix H7 (Fig. 2b), and the RNA binding site of TuMV CP includes the universally conserved pocket in flexible filamentous plant viruses formed by amino acids Ser, Arg, and Asp (Supplementary Fig. 2c) (4,6. $^{4}$

For TuMV VLPs initial cryoEM results imposing helical symmetry did not converge in reproducible 3D maps (data not shown), thus, a 3D classification of the filament segments was performed without any imposed symmetry. The results (Supplementary Fig. 3 ) revealed that only about $60 \%$ of the particles display clear helical arrangement with well defined CP subunits (classes 1 and 3 in Supplementary Fig. 3a,c), while the rest of the groups show 3D maps with poor structural features and no indication of well ordered helical arrangement (Supplementary Fig. 3b,d-f). Thus, the absence of ssRNA in the VLPs produces labile multimers with distorted local regions along the filaments. This classification did not detect any population of VLPs constructed by stacked rings.

The two groups of VLP segments with good helical features (classes 1 and 3) were further refined to 3D maps with final resolutions about $8 \AA$ (Supplementary Fig. 4). This poor definition compared with the results for TuMV virions, suggests that VLPs are less stable, structurally more heterogeneous, and hence their 3D averages are limited in structural details. At this level of resolution it is not possible to build accurate atomic models. Both groups, however, exhibit helical symmetry parameters (Supplementary Fig. 4) identical to that of the TuMV 
a
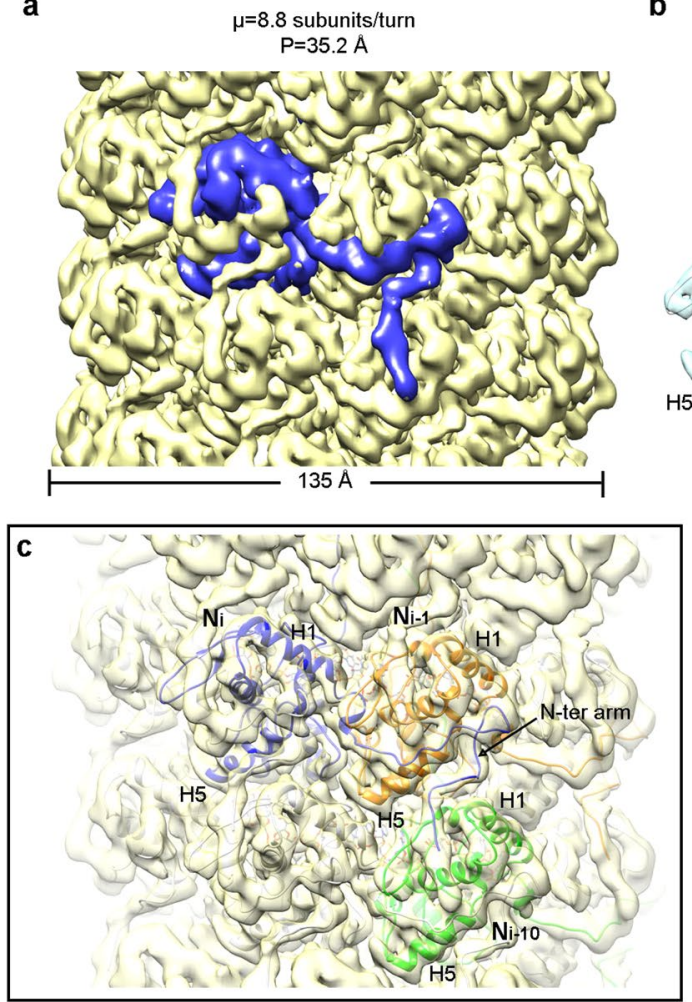

b
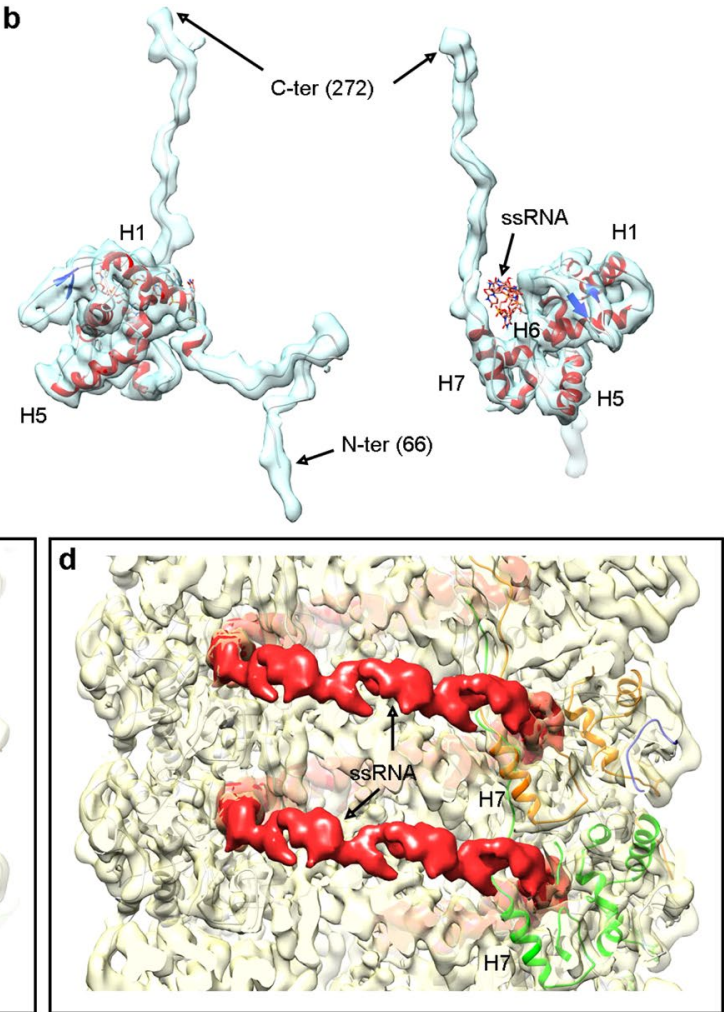

Figure 2. CryoEM 3D structure of TuMV. (a) Rendering of the 3D map calculated for TuMV virions (yellow). The density for one of the CP subunits is depicted blue. Helical symmetry parameters are indicated: $\mu$ stands for the number of subunits per turn of the helix; and $\mathrm{P}$ for the helical pitch. (b) Semitransparent representation of the density attributed to a single TuMV CP, together with the modeled atomic coordinates and a polyU that represent the ssRNA. Two different orientations are shown. (c) The cryoEM map for TuMV is seen semitransparent together with the fitted structures for several CP subunits displayed in different colors. (d) Cutaway view of TuMV cryoEM map with ribbons from the fitted coordinates for some CP subunits. The isolated density for the ssRNA is seen in solid mode and red colored. Along the panels some $\alpha$-helices of the atomic structure for TuMV CP are labeled (H1, H5, H6, and H7).

virions (Fig. 2), thus, we assume that the overall organization of the virions is kept in the VLPs despite the lack of nucleic acid. For the interpretation of the structures for VLPs, we fit the atomic coordinates modeled for TuMV virions (a polymer of $20 \mathrm{CP}$ subunits) as a rigid body. In the cryoEM maps for both groups of VLP segments, the helical path for the ssRNA derived from TuMV virions (the atoms for the nucleic acid were not included in the rigid body fitting) resides in an empty passage (Fig. 3a and Supplementary Fig. 5a). This confirms the absence of the ssRNA in the VLPs and that the fitting of the CP multimer is on the correct register with respect to the 3D maps. In class 1, helix H7, that delimits the ssRNA binding groove in the virions (Fig. 2d), seems to move towards the inner side of the filament (Fig. 3a). The fitting of the coordinates for the oligomer of CPs lefts the N-terminal arm outside the density: fully outside in class 1 (Fig. 3b); or only in the last region that participates in axial interactions in class 3 (Supplementary Fig. 5b). Also, the densities for helices H1 and H5 are incomplete, and both secondary structure elements stick out at certain degree from the cryoEM maps (Fig. $3 \mathrm{~b}$ and Supplementary Fig. 5b). Thus, the role of the $\mathrm{N}$-terminal arm in polymerization and the position of helices $\mathrm{H} 1$ and $\mathrm{H} 7$ are perturbed in the absence of the ssRNA. To gain some insights into the influence of the ssRNA over these structural elements we revisit the atomic model for TuMV virions (Fig. 3c). In the boundary between CP subunits there is a network of protein-RNA and protein-protein interactions that supports the proper orientation of the flexible N-terminal arm. Residue N103 from one CP subunit $\left(\mathrm{N}_{\mathrm{i}}\right)$, and the pair R204 and R209 from the adjacent $\mathrm{CP}\left(\mathrm{N}_{\mathrm{i}-1}\right)$ interact with the phosphate backbone of the ssRNA (Fig. 3d). At the same time, these two regions are connected between them, in such a way that R204 interacts with the beginning of the N-terminal arm that contains the aforementioned N103, and S102 and T104 at the neighboring subunit (Fig. 3c). These local interactions with the ssRNA and between $\mathrm{CPs}$ serve to anchor helix $\mathrm{H} 1$ and the $\mathrm{N}$-terminal arm of one $\mathrm{CP}$ subunit $\left(\mathrm{N}_{\mathrm{i}}\right)$ and helices $\mathrm{H} 5$ and $\mathrm{H} 6$ of the neighbor $\left(\mathrm{N}_{\mathrm{i}-1}\right)$. Since helix $\mathrm{H} 5$ builds part of the groove for the interaction with the $\mathrm{N}$-terminal arm, the contacts with the ssRNA modulate both the donor and the acceptor in the interaction via the N-terminal arm. The three residues that make direct contact with the ssRNA in this region are highly conserved in potyviruses (N103 90\%, R204 80\%, and R204 83\%) and are also seen involved in the same interactions with the nucleic acid in $\mathrm{WMV}^{4}$ and $\mathrm{PVY}^{5}$. In this same local region, helix $\mathrm{H} 1$ and the $\mathrm{N}$-terminal arm (subunit $\mathrm{N}_{\mathrm{i}}$ ) interact with the $\mathrm{N}$-terminal arm of other subunit from the next helical turn $\left(\mathrm{N}_{\mathrm{i}-9}\right.$ in Fig. 3c). Here, the hydrophobic interaction F115-Y80 (Fig. 3e) and the salt bridge E97-R76 (Fig. 3f) are key to set the $90^{\circ}$ turn of the N-terminal arm towards 

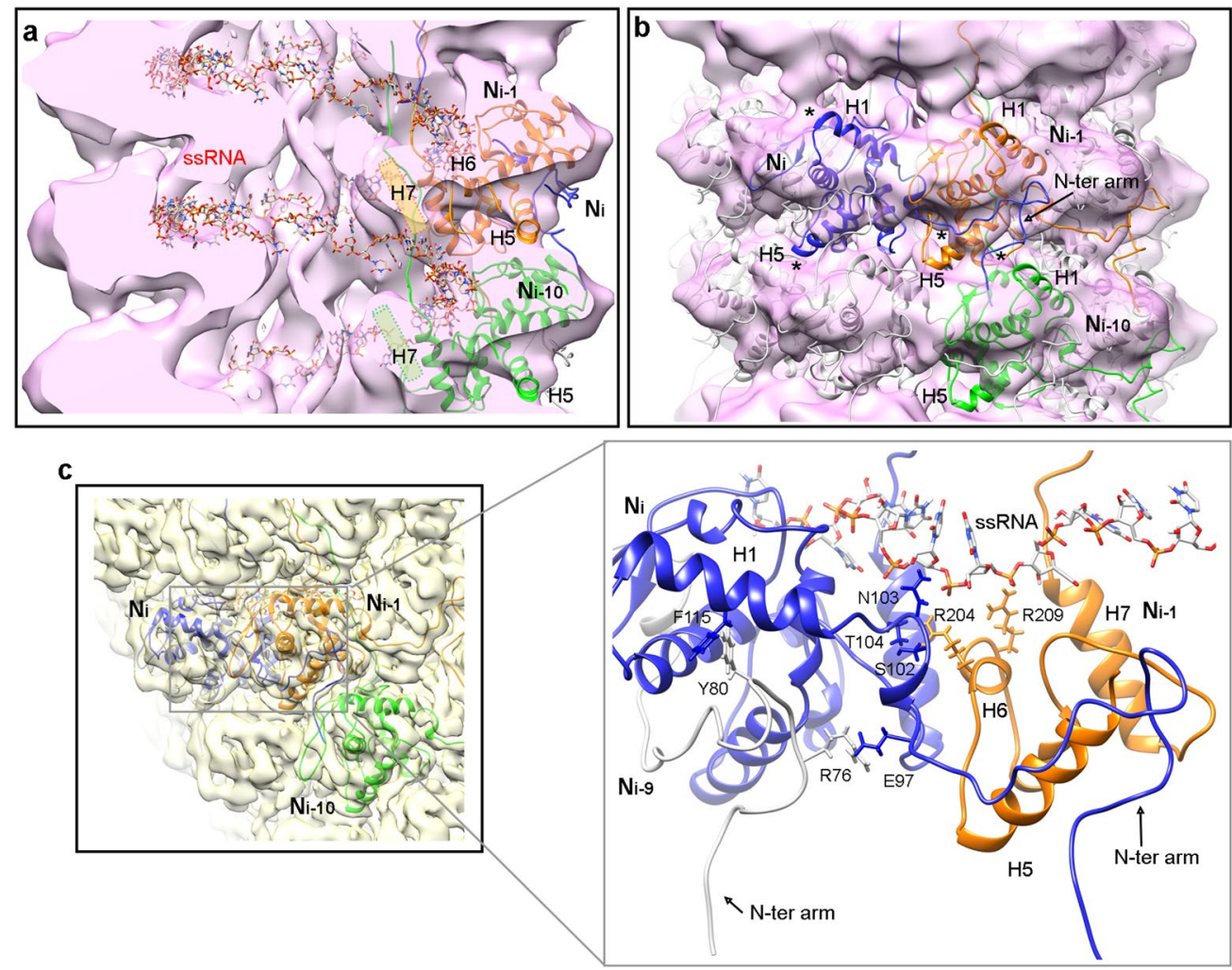

d

e
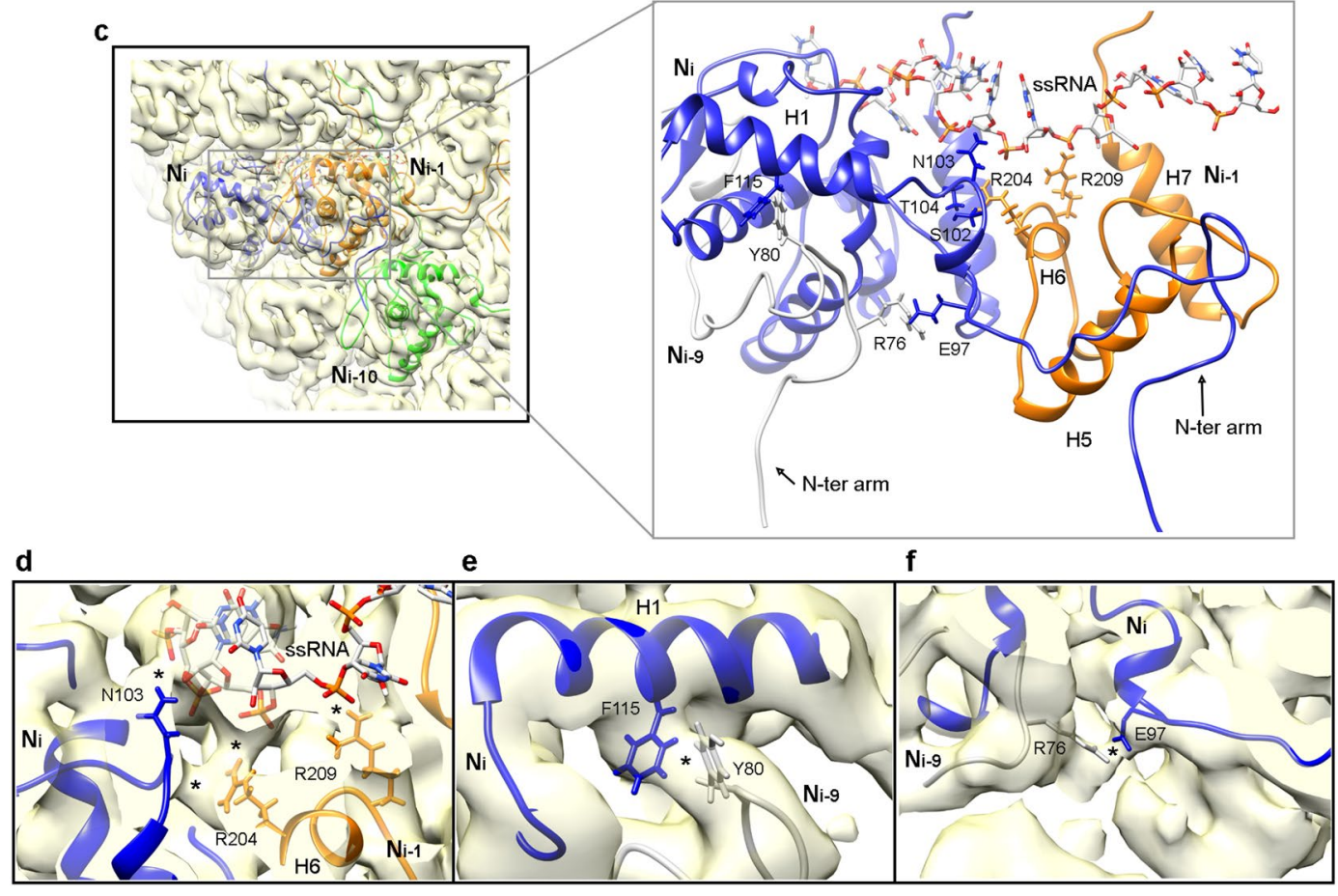

f

Figure 3. Structure of VLPs and the role of CP-RNA interactions. (a) Cut-away rendering of the cryoEM map for class 1 of TuMV VLP. Atomic models for several TuMV CPs and the ssRNA are also included. After rigid body fitting of the coordinates derived from TuMV virion, the ssRNA runs in an empty channel. The position of helix $\mathrm{H} 7$ seem to have moved in the VLP towards the inner side of the filament, and the new putative location is indicated by cylinders. (b) The fitted coordinates for the multimer of TuMV CPs are seen inside the semitransparent map for class 1 of TuMV VLP. Regions of the atomic models that lie outside the density are labeled with asterisks in subunit $\mathrm{N}_{\mathrm{i}}$. (c) Protein-RNA and protein-protein interactions at the interface between CP subunits in TuMV virions. Three CP subunits are depicted, together with the ssRNA. Residues that participate in protein-RNA and/or protein-protein interactions are indicated. Some regions of CP subunit $\mathrm{N}_{\mathrm{i}-1}$ are not displayed for clarity. The thumbnail at the left shows the orientation. (d-f) Close-up views of the cryoEM map and atomic coordinates for TuMV virions focused on the regions of protein-protein and protein-RNA interactions. The contacts between residues (labeled with asterisks) are visible in the $3 \mathrm{D}$ density map at $2 \sigma$ (panels $\mathrm{d}, \mathrm{e}$ ) or $1 \sigma$ density thresholds. In the panels some $\alpha$-helices of the atomic structure for TuMV CP are labeled.

the next turn of the helix. The F115-Y80 connection between TuMV CPs has equivalent pairs in WMV and PVY, where the hydrophobic pair is established between Tyr and Val residues. However, the E97-R76 salt bridge has no counterparts in the other two potyviruses, probably due the high diversity of sequences at the $\mathrm{N}$-terminal arm.

As opposed to icosahedral viruses, in helical viruses the genetic material is bound to copies of the viral nucleoprotein or $\mathrm{CP}$ along the entire genomic length, and each nucleoprotein subunit interacts with the genome. Thus, the absence of the nucleic acid in VLPs is expected to modify the entire structure. Interestingly, the VLPs in the current work keep the helical symmetry of the virions, while PVY VLPs derived from overpexpressed CP subunits in E.coli arrange in the form of stacked rings of 8 subunits. Although at lower resolution, VLPs from Alternanthera mosaic virus (AltMV, a potexvirus) produced in vitro were seen in helical arrangement ${ }^{14}$. These differences in the architecture of VLP assemblies need to be further explored for the design of nanoparticles based on CPs from flexible filamentous plant viruses. The helical arrangement of TuMV VLPs allows the comparison of their structure with TuMV virions, and shows that the interaction with the ssRNA in between subunits govern 
the network of contacts between CPs mediated by N-terminal arms that play as molecular staples, and that these interactions are lost in the absence of the nucleic acid.

\section{Materials and Methods}

Purification of TuMV virions and VLPs. TuMV (isolate UK 1) ${ }^{16}$ was propagated in plants of Indian mustard (Brassica juncea), which were harvested 30 days post-inoculation. For VLP production, five-week old Nicotiana benthamiana plants were agroinfiltrated for CP transient expression. Agrobacterium tumefaciens (LBA4404 strain) transformed with the CP construction was subcultured and grown overnight, pelleted at $2000 \times g$, resuspended to $\mathrm{OD}_{600}=1.2$ in MMA buffer ( $10 \mathrm{mM}$ MES, pH 5.6; $10 \mathrm{mM}$ magnesium chloride; $450 \mu \mathrm{M}$ acetosyringone), and then infiltrated into the leaves using a blunt-ended $2 \mathrm{~mL}$ syringe. Tissue was harvested $10-12$ days post-agroinfiltration. VNPs were purified either from Indian mustard (150 g) or N. benthamiana $(100 \mathrm{~g})$ plant material as described ${ }^{10}$. Briefly, plant tissue was finely ground in $0.5 \mathrm{M}$ potassium phosphate $\mathrm{pH} 7.5,1: 2(\mathrm{w} / \mathrm{v})$ in an electrical tissue grinder, at $4^{\circ} \mathrm{C}$. The resulting suspension was extracted with one volume of chloroform at $4{ }^{\circ} \mathrm{C}$. Phases were separated by centrifugation; aqueous phase was filtered through Miracloth. After that, VNPs were precipitated with 6\% PEG $6000(\mathrm{w} / \mathrm{v}), 4 \% \mathrm{NaCl}(\mathrm{w} / \mathrm{v})$. They were allowed to precipitate for $90 \mathrm{~min}$ at $4{ }^{\circ} \mathrm{C}$. The particles were recovered by centrifugation for $10 \mathrm{~min}$ at $12,000 \times \mathrm{g}$. The pellet was resuspended overnight in $0.5 \mathrm{M}$ potassium phosphate $\mathrm{pH} 7.5,10 \mathrm{mM}$ EDTA. The solution was clarified by centrifugation $(10 \mathrm{~min}$ at $9000 \times \mathrm{g})$ and the VNPs pelleted $(2 \mathrm{~h}$ at $80000 \times \mathrm{g}$ ). The pellet was resuspended in $0.25 \mathrm{M}$ potassium phosphate $\mathrm{pH} 7.5,10 \mathrm{mM}$ EDTA, and $\mathrm{CsCl}$ was added to a final density of $1.27 \mathrm{~g} / \mathrm{cm}^{3}$. The resulting solution was subjected to centrifugation at $150000 \times \mathrm{g}$ for $18 \mathrm{~h}$ at $4^{\circ} \mathrm{C}$. A visible band in the gradient containing the particles was recovered by punching the tube with syringe and needle. It was diluted in $0.25 \mathrm{M}$ potassium phosphate $\mathrm{pH} 7.5,10 \mathrm{mM}$ EDTA and pelleted by centrifugation $(2 \mathrm{~h}$ at $80000 \times \mathrm{g})$. Finally, the pellet was resuspended in $50 \%$ glycerol $(\mathrm{v} / \mathrm{v}), 5 \mathrm{mM}$ Tris $\mathrm{pH} 7.5$, $5 \mathrm{mM}$ EDTA at a final concentration of $1 \mathrm{mg} / \mathrm{ml}$, and stored at $-20^{\circ} \mathrm{C}$ until further use. VNP concentration was determined spectrophotometrically considering an absorption coefficient $\left(\mathrm{A}_{0.1 \%, 1 \mathrm{~cm}}\right.$ at $\left.260 \mathrm{~nm}\right)$ of 2.65.

CryoEM and image processing. TuMV VNPs solutions were applied to Quantifoil R2/2 holey carbon grids covered with a thin carbon layer, and the cryoEM grids were prepared using a Vitrobot (FEI). Movie frames images were collected in a Titan Krios FEI electron microscope operated at $300 \mathrm{kV}$ using a K2 direct detector (GATAN). Nominal magnification was of x130.000 for both TuMV virions and VLPs, resulting in a sampling of $1.1 \AA$ /pixel. The micrographs were taken during $9 \mathrm{~s}$ exposures in electron counting mode producing movies with 40 frames and a total dose of $40 \mathrm{e}^{-} / \AA^{2}$. Motion between frames was corrected using frames 3 to 31 , resulting in accumulative dose of $31 \mathrm{e}^{-} / \AA^{2}$. The contrast transfer function of the micrographs for both samples was estimated using CTFFIND ${ }^{17}$. In the corrected micrographs filaments were manually selected in Relion $2^{18}$, resulting in 444,678 overlapping segments (boxes of 250 pixels $\times 250$ pixels, with a step of 8 pixels between segments) for TuMV virions and in 307,333 segments (boxes of 200 pixels $\times 200$ pixels, with a step of 8 pixels between segments) for TuMV VLPs. CryoEM density maps were calculated in Relion2. First, 2D classification and particle sorting allowed us to isolate sets of good-quality filament segments $(335,228$ segments for TuMV virions, and 118,597 for VLPs). For 3D refinement of TuMV virions, the initial reference was a cylinder with the diameter of the filaments. Starting with such featureless structure required to re-run the $3 \mathrm{D}$ refinement using the output from the first refinement as the new reference for a second round. Local optimization of twist and rise was carried out during $3 \mathrm{D}$ refinements. 3D classification with local searches of symmetry resulted in three classes with different, although very similar, symmetry values. Helical symmetry in real space was imposed to the final map to obtain homogeneity among the asymmetric units for molecular modeling. In the other hand, for TuMV VLPs the density map for TuMV virions low-pass filtered at $40 \AA$ was used as initial reference map. Several 3D classifications were carried out, but only the one performed without imposing symmetry segregated classes with clear helical symmetry from distorted segments. Helical symmetry was imposed during the 3D refinement of selected classes and to their final 3D cryoEM maps.

Atomic model building for TuMV CP. The atomic structure for WMV CP (pdb code 5ODV) ${ }^{4}$ was used as a template for the atomic model building of TuMV CP. Density for a TuMV CP subunit was isolated from the 3D cryoEM map by segmentation using the Segger ${ }^{19}$ method in Chimera ${ }^{20}$, which was also used to produce figures and movies. The amino acid sequence of WMW CP was mutated manually using $\operatorname{Coot}^{21}$ to match the sequence for TuMV CP. Further modeling of the TuMV CP atomic structure was carried out manually using $\operatorname{Coot}^{21}$ and the stereochemistry of the model was improved by real-space refinement in Phenix ${ }^{22}$. When the final atomic coordinates for TuMV CP are compared with the structure of WMV CP, the rmsd between their C-alpha backbone is just $1.2 \AA$ in the modeled region. For the ssRNA, a modeled polyU was included in the refinement. To build up a multimer of CPs and the ssRNA a final refinement in Phenix was performed using noncrystallographic symmetry. Evaluation of the modeled atomic structure for TuMV CP was carried out in MolProbity ${ }^{23}$. To calculate the surface electrostatic potential for the atomic structure of TuMV CP, the atomic coordinates were loaded in Bluues server (http://protein.bio.unipd.it/bluues) ${ }^{24}$ and the potential was determined based on generalized Born radii $^{25}$.

Received: 5 July 2019; Accepted: 7 October 2019;

Published online: 28 October 2019

\section{References}

1. Kendall, A. et al. Structure of flexible filamentous plant viruses. J Virol 82, 9546-54 (2008).

2. DiMaio, F. et al. The molecular basis for flexibility in the flexible filamentous plant viruses. Nat Struct Mol Biol 22, 642-4 (2015).

3. Agirrezabala, X. et al. The near-atomic cryoEM structure of a flexible filamentous plant virus shows homology of its coat protein with nucleoproteins of animal viruses. Elife 4, e11795 (2015).

4. Zamora, M. et al. Potyvirus virion structure shows conserved protein fold and RNA binding site in ssRNA viruses. Sci Adv 3, eaao2182 (2017) 
5. Kezar, A. et al. Structural basis for the multitasking nature of the potato virus Y coat protein. Sci Adv 5, eaaw3808 (2019).

6. Valle, M. Structural Homology Between Nucleoproteins of ssRNA Viruses. Subcell Biochem 88, 129-145 (2018).

7. Steele, J. F. C. et al. Synthetic plant virology for nanobiotechnology and nanomedicine. Wiley Interdiscip Rev Nanomed Nanobiotechnol 9 (2017).

8. Eiben, S. et al. Plant virus-based materials for biomedical applications: Trends and prospects. Adv Drug Deliv Rev (2018).

9. Lee, K. L., Uhde-Holzem, K., Fischer, R., Commandeur, U. \& Steinmetz, N. F. Genetic engineering and chemical conjugation of potato virus X. Methods Mol Biol 1108, 3-21 (2014).

10. Sanchez, F. \& Ponz, F. Presenting Peptides at the Surface of Potyviruses In Planta. Methods Mol Biol 1776, 471-485 (2018).

11. Balke, I. \& Zeltins, A. Use of plant viruses and virus-like particles for the creation of novel vaccines. Adv Drug Deliv Rev (2018).

12. Kushnir, N., Streatfield, S. J. \& Yusibov, V. Virus-like particles as a highly efficient vaccine platform: diversity of targets and production systems and advances in clinical development. Vaccine 31, 58-83 (2012).

13. Yuste-Calvo, C., González-Gamboa, I., Pacios, L. F., Sánchez, F. \& Ponz, F. Structure-Based Multifunctionalization of Flexuous Elongated Viral Nanoparticles. ACS Omega 4, 5019-5028 (2019).

14. Donchenko, E. K. et al. Structure and properties of virions and virus-like particles derived from the coat protein of Alternanthera mosaic virus. PLoS One 12, e0183824 (2017).

15. Gonzalez-Gamboa, I., Manrique, P., Sanchez, F. \& Ponz, F. Plant-made potyvirus-like particles used for log-increasing antibody sensing capacity. J Biotechnol 254, 17-24 (2017).

16. Sánchez, F., Wang, X., Jenner, C. E., Walsh, J. A. \& Ponz, F. Strains of Turnip mosaic potyvirus as defined by the molecular analysis of the coat protein gene of the virus. Virus Research 94, 33-43 (2003).

17. Mindell, J. A. \& Grigorieff, N. Accurate determination of local defocus and specimen tilt in electron microscopy. J Struct Biol 142, 334-47 (2003).

18. He, S. \& Scheres, S. H. Helical reconstruction in RELION. J Struct Biol (2017).

19. Pintilie, G. D., Zhang, J., Goddard, T. D., Chiu, W. \& Gossard, D. C. Quantitative analysis of cryo-EM density map segmentation by watershed and scale-space filtering, and fitting of structures by alignment to regions. J Struct Biol 170, 427-38 (2010).

20. Pettersen, E. F. et al. UCSF Chimera-a visualization system for exploratory research and analysis. J Comput Chem 25, 1605-12 (2004).

21. Emsley, P., Lohkamp, B., Scott, W. G. \& Cowtan, K. Features and development of Coot. Acta Crystallogr D Biol Crystallogr 66, 486-501 (2010)

22. Adams, P. D. et al. PHENIX: a comprehensive Python-based system for macromolecular structure solution. Acta Crystallogr D Biol Crystallogr 66, 213-21 (2010).

23. Chen, V. B. et al. MolProbity: all-atom structure validation for macromolecular crystallography. Acta Crystallogr D Biol Crystallogr 66, 12-21 (2010).

24. Walsh, I. et al. Bluues server: electrostatic properties of wild-type and mutated protein structures. Bioinformatics 28, 2189-90 (2012).

25. Fogolari, F. et al. Bluues: a program for the analysis of the electrostatic properties of proteins based on generalized Born radii. $B M C$ Bioinformatics 13(Suppl 4), S18 (2012).

\section{Acknowledgements}

This research was partially funded by grants BFU2015-66326-P and PGC2018-098996-B-I00 (Spanish Ministry of Science, Innovation and Universities) to MV, RTA2015-00017 (INIA) and APCIN2016-00014-00-00 (Nanobioagri, ArimNet 2) to FP. We thank the Spanish Ministry of Science for the Severo Ochoa Excellence Accreditations to the CIC bioGUNE (SEV-2016-0644) and to the CBGP (SEV-2016-0672). We also thank Lucía Zurita for technical assistance. We thank the Netherlands Centre for Electron Nanoscopy, NeCEN (Leiden, The Netherlands) for the collection of cryoEM images at the facility performed by Dr. Ludovich Renault. The cryoEM maps for TuMV virions and VLPs, and the calculated atomic model for TuMV CP are available in the Electron Microscopy Data Bank (www.emdatabank.org) and the Protein Data Bank (www.rcsb.org) under accession codes EMD-10373, EMD-10374, and 6T34 respectively.

\section{Author contributions}

M.V. and F.P. designed the experiments. C.Y. and F.S. prepared TuMV samples, R.C. and D.G. the cryoEM grids. R.C. and M.V. performed the cryoEM image processing and calculated the atomic model. M.V. wrote the manuscript with contributions from all the authors.

\section{Competing interests}

The authors declare no competing interests.

\section{Additional information}

Supplementary information is available for this paper at https://doi.org/10.1038/s41598-019-51823-4.

Correspondence and requests for materials should be addressed to M.V.

Reprints and permissions information is available at www.nature.com/reprints.

Publisher's note Springer Nature remains neutral with regard to jurisdictional claims in published maps and institutional affiliations.

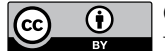

Open Access This article is licensed under a Creative Commons Attribution 4.0 International

License, which permits use, sharing, adaptation, distribution and reproduction in any medium or format, as long as you give appropriate credit to the original author(s) and the source, provide a link to the Creative Commons license, and indicate if changes were made. The images or other third party material in this article are included in the article's Creative Commons license, unless indicated otherwise in a credit line to the material. If material is not included in the article's Creative Commons license and your intended use is not permitted by statutory regulation or exceeds the permitted use, you will need to obtain permission directly from the copyright holder. To view a copy of this license, visit http://creativecommons.org/licenses/by/4.0/.

(c) The Author(s) 2019 